\title{
Design of Yagi Antenna with Slow-Wave Half-Mode SIW Feeding Technique for Ku Band Applications
}

\author{
Bo Han, ${ }^{1,2}$ Shibing Wang, ${ }^{1}$ Jia Zhao, ${ }^{1}$ and Xiaofeng Shi ${ }^{1}$ \\ ${ }^{1}$ School of Computer and Information Engineering, Fuyang Normal University, Fuyang 236037, China \\ ${ }^{2}$ State Key Laboratory of Millimeter Waves, Southeast University, Nanjing 210096, China \\ Correspondence should be addressed to Bo Han; hanbo315315@163.com
}

Received 5 July 2017; Revised 20 September 2017; Accepted 25 October 2017; Published 12 December 2017

Academic Editor: Yu Jian Cheng

Copyright ( 2017 Bo Han et al. This is an open access article distributed under the Creative Commons Attribution License, which permits unrestricted use, distribution, and reproduction in any medium, provided the original work is properly cited.

\begin{abstract}
A novel planar Yagi antenna printed on a microwave substrate with dielectric constant of 3.55 for Ku band applications has been presented in this paper. The proposed antenna has been fed by the slow-wave half-mode substrate-integrated waveguide and has achieved good characteristics, such as reduced size, high gain, broadband, and low insertion loss. The proposed antenna has been fabricated by Rogers 4350 substrate with lengths of two arms for dipole $0.46 \lambda_{0}$. Measured results indicate that the impedance bandwidth (below $-10 \mathrm{~dB}$ return loss) is from $15.4 \mathrm{GHz}$ to $19.4 \mathrm{GHz}$ with peak gain $7.49 \mathrm{dBi}$. Both simulations and experiments convince that the proposed antenna could have reliable applications for Ku band wireless communications.
\end{abstract}

\section{Background and Introduction}

The planar Yagi antenna becomes a popular opportunity for the design of wireless communication system as advantages of low complexity, low cost, low profile, lightweight, and easy integration. Many researchers have done numerous study works on the design of Yagi antenna. Practically, the feeding system plays an important role in the performance of the Yagi antenna. The quasi-Yagi antenna and array fed by the microstrip-to-CPS balun have been proposed in $[1,2]$, respectively. Extremely wide frequency bandwidth and good radiation characteristics have been achieved by this feeding technique. The substrate-integrated waveguide (SIW) technologies have been proposed to combine the advantages of rectangular waveguide and microstrip circuits [3, 4]. Substrate-integrated folded waveguides (SIFW) and halfmode substrate-integrated waveguide (HMSIW) have been proposed to reduce the size of SIW in $[5,6]$, respectively. The design of a miniaturized substrate-integrated waveguide using embedded split-ring resonators has been illustrated in $[7,8]$. Yagi antennas fed by SIW and HMSIW have been presented in $[9,10]$, in which high gain and broadband have achieved with a simple structure. Recently, the slow-wave substrate-integrated waveguide (SW-SIW) by a double- layer substrate with the bottom layer including metallized via-holes connected to the ground has been proposed in [11]. Compared with the classical SIW, the SW-SIW can reduce the longitudinal dimension by more than $40 \%$ as the phase velocity is significantly smaller than the SIW structure.

In the paper, a novel planar Yagi antenna fed by the slowwave half-mode SIW (SW-HMSIW) has been proposed for $\mathrm{Ku}$ band applications. The proposed antenna has been fabricated by a conventional PCB process with compact size $21.1 \times 13.4 \times 1.016 \mathrm{~mm}^{3}$. Measured results show that the impedance bandwidth is from $15.4 \mathrm{GHz}$ to $19.4 \mathrm{GHz}$ with an average gain of more than $8.1 \mathrm{dBi}$.

This paper is organized as follows. Section 2 describes the design of the proposed SW-HMSIW and SW-HMSIW-fed planar Yagi antenna. Section 3 presents the electromagnetic simulations and experimental results of the proposed Yagi antennas. Finally, the summary and conclusion are given in Section 4.

\section{Design of Proposed SW-HMSIW-Fed Yagi Antenna}

In this section, the design of slow-wave SIW and SWHMSIW-fed Yagi antenna is illustrated in detail. 


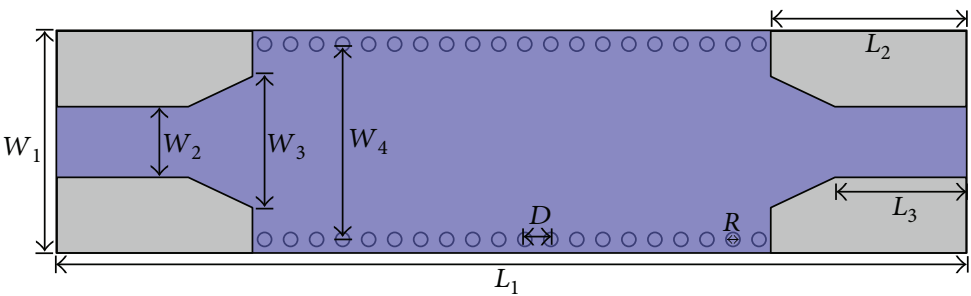

(a)

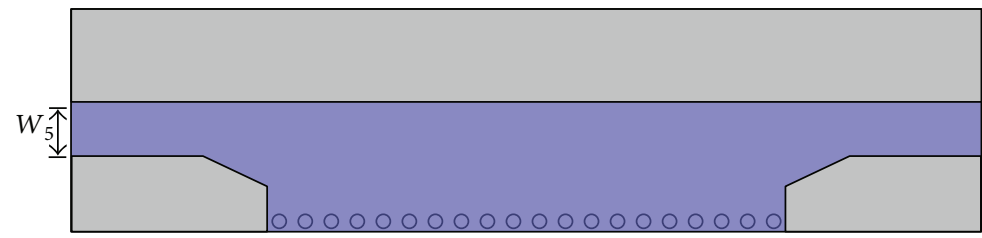

(b)

Figure 1: Substrate-integrated waveguide layout fabricated by Rogers 4350 substrate. (a) Substrate-integrated waveguide; (b) half-mode substrate-integrated waveguide.

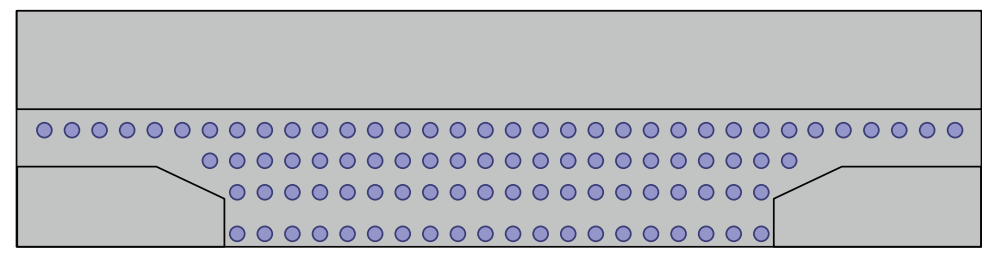

(a)

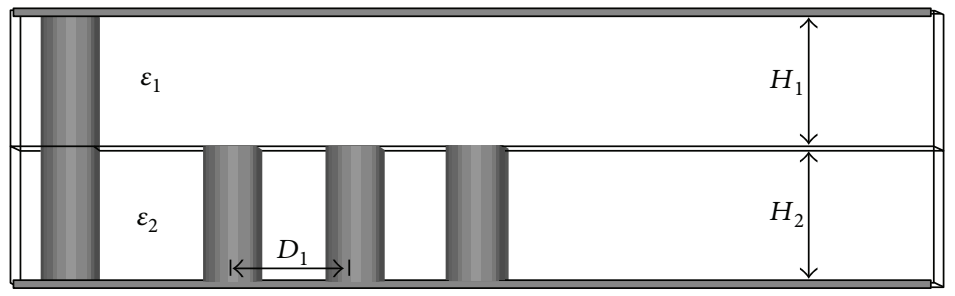

(b)

FIgURE 2: Slow-wave substrate-integrated waveguide layout fabricated by Rogers 4350 substrate. (a) Top view; (b) cross-section view.

2.1. Design of Slow-Wave SIW. The layouts of the substrateintegrated waveguide and half-mode substrate-integrated waveguide have been shown in Figure 1, where $W_{4}$ represents the distance between the two rows of vias and $H$ represents the height of the Rogers 4350 dielectric substrate. For the Rogers 4350 substrate, the relative permittivity $\varepsilon_{\mathrm{r}}$ is 3.45 , the value of tangent for the loss angle is 0.004 , and the thickness of the metal is 50 microns. The diameter of the metallized via in the dielectric substrate $R$ is 0.4 millimeters, and the center distance between the metallized vias $D$ is 0.8 millimeters.

The layout of the slow-wave half-mode substrateintegrated waveguide with the same geometry size has been shown in Figure 2. There are two layers of dielectric substrate which are made up of Rogers 4350 substrate with diameter of the metallized via 0.4 millimeters for the SW-HMSIW structure. In order to realize slow-wave effect, three rows of metallized vias as shown in Figure 2(b) are designed in medium 2. The bottoms of these rows' metallized vias are connected
TABLE 1: Values of substrate-integrated waveguide geometry parameters designed on Rogers 4350 substrate (all units in millimeters).

\begin{tabular}{lccc}
\hline Parameters & Values & Parameters & Values \\
\hline$W_{1}$ & 6.8 & $L_{1}$ & 28 \\
$W_{2}$ & 2.2 & $L_{2}$ & 6 \\
$W_{3}$ & 4 & $L_{3}$ & 4 \\
$W_{4}$ & 6 & $W_{5}$ & 1.76 \\
$R$ & 0.4 & $D$ & 0.8 \\
$H_{1}$ & 0.508 & $D_{1}$ & 0.9 \\
$H_{2}$ & 0.508 & & \\
\hline
\end{tabular}

to the ground while the tops are suspended. The value of the central distance between adjacent two rows of metallized vias $D_{1}$ is 0.9 millimeters. All values of physical dimensions for these substrate-integrated waveguides designed on Rogers 4350 substrate have been shown in Table 1 . 


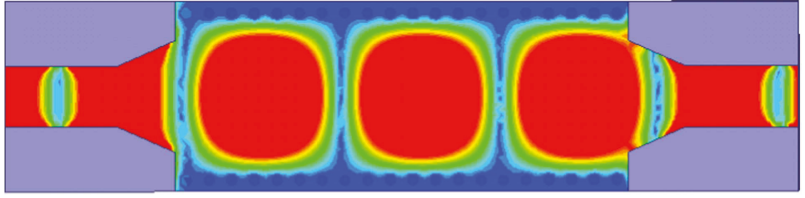

(a)

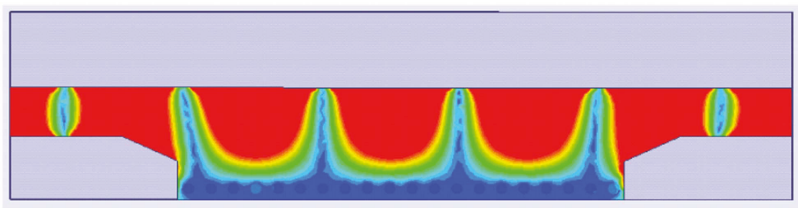

(b)

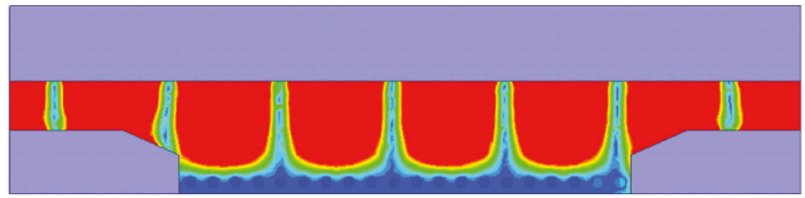

(c)

FIGURE 3: Simulated surface E-filed distribution of substrateintegrated waveguides at $20 \mathrm{GHz}$. (a) Substrate-integrated waveguide; (b) half-mode substrate-integrated waveguide; (c) slow-wave half-mode substrate-integrated waveguide.

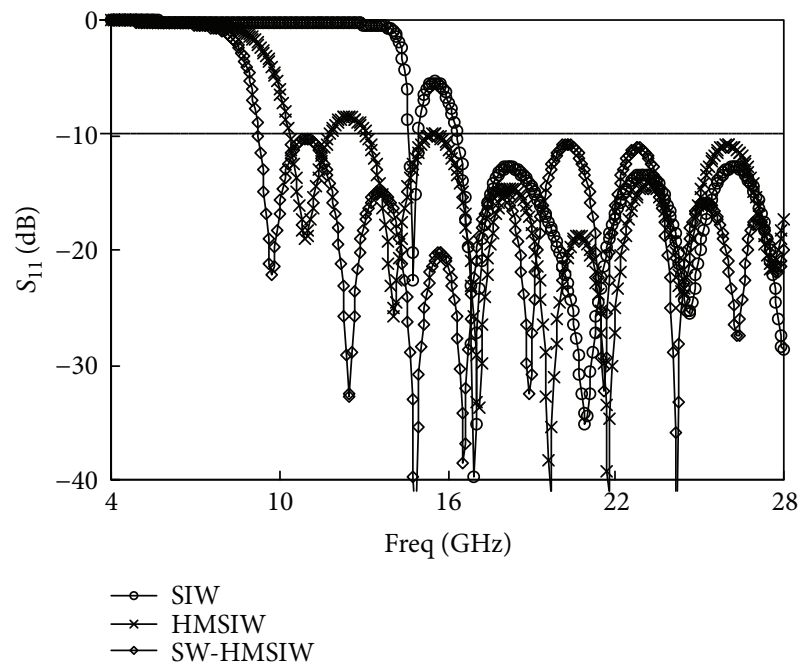

Figure 4: Simulated $\left|S_{11}\right|$ parameters of different mode substrateintegrated waveguides.

The proposed substrate-integrated waveguides have been computed by Ansoft's HFSS software, and the simulation port is set as a lumped port [12]. The simulated surface E-filed distributions for the SIW, HMSIW, and SWHMSIW at $20 \mathrm{GHz}$ have been shown in Figure 3. It can be seen that three propagation cycles of $\mathrm{E}$ filed are illustrated for the SIW and HMSIW. However, the number of the E-filed propagation cycle is four for SW-HMSIW. Therefore, the slow-wave effect can be well explained graphically.

Figure 4 shows the simulated scattering parameters for the different mode substrate-integrated waveguides. According to this diagram, the cutoff frequency of HMSIW is

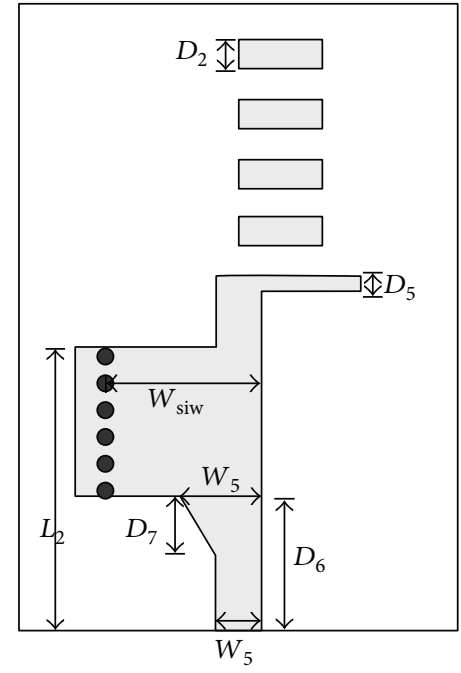

(a)

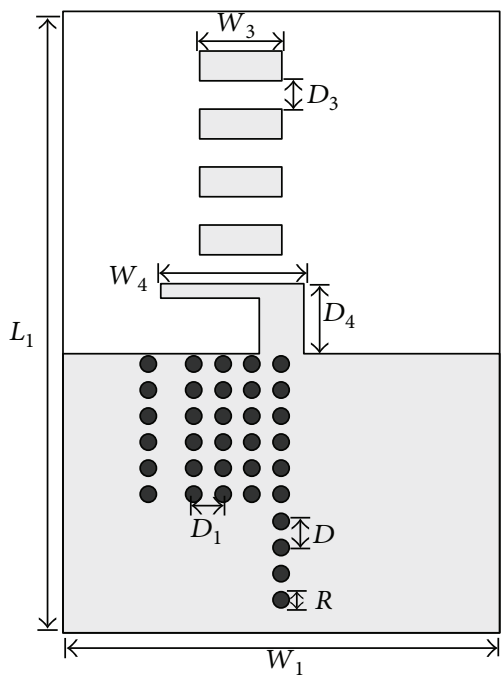

(b)

FIgURE 5: Layout of the proposed slow-wave half-mode substrateintegrated waveguide Yagi antenna designed on Rogers 4350 substrate. (a) Top view; (b) bottom view.

10.35 GHz. Compared to the structure HMSIW, the cutoff frequency of the SW-HMSIW is reduced by $1.2 \mathrm{GHz}$. Hence, a narrower waveguide could be obtained when an SWHMSIW is used instead of a conventional HMSIW for the same targeted cutoff frequency [9].

2.2. Design of SW-HMSIW-Fed Yagi Antenna. The layout of the proposed slow-wave half-mode substrate-integrated waveguide Yagi antenna which consists of two layers of Rodgers 4350 substrate has been plotted in Figure 5. In the top view, there are a truncated metal plane acted as the top layer of the SW-HMSIW, one of the parallel lines, one arm of the dipole antenna, and four director elements. A row of metallized vias which connect the top metal and ground metal through two dielectric substrates acts as the sidewall of the SW-HMSIW. The bottom substrate consists 
TABLE 2: Values of the geometry parameters for the proposed Yagi antenna (all units in millimeters).

\begin{tabular}{lccc}
\hline Parameters & Values & Parameters & Values \\
\hline$W_{1}$ & 13.4 & $L_{1}$ & 21.1 \\
$W_{\text {sim }}$ & 2.2 & $D_{2}$ & 1 \\
$W_{3}$ & 2.5 & $L_{2}$ & 9.54 \\
$W_{4}$ & 4.4 & $W_{5}$ & 1.4 \\
$R$ & 0.4 & $D$ & 0.8 \\
$H_{1}$ & 0.508 & $D_{1}$ & 0.8 \\
$H_{2}$ & 0.508 & $D_{3}$ & 1 \\
$D_{4}$ & 1.9 & $D_{5}$ & 0.5 \\
$D_{6}$ & 4.5 & $D_{7}$ & 2 \\
$W_{6}$ & 2.5 & & \\
\hline
\end{tabular}

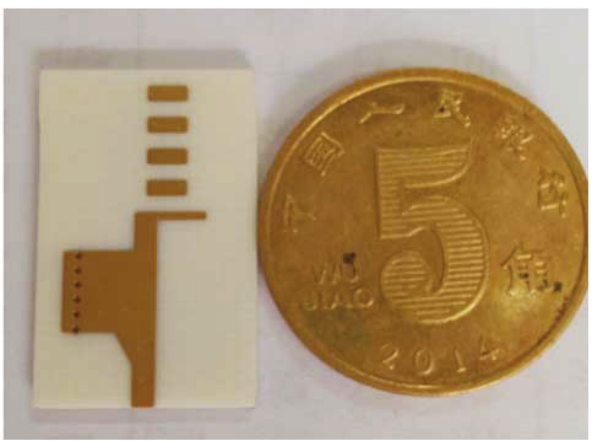

(a)

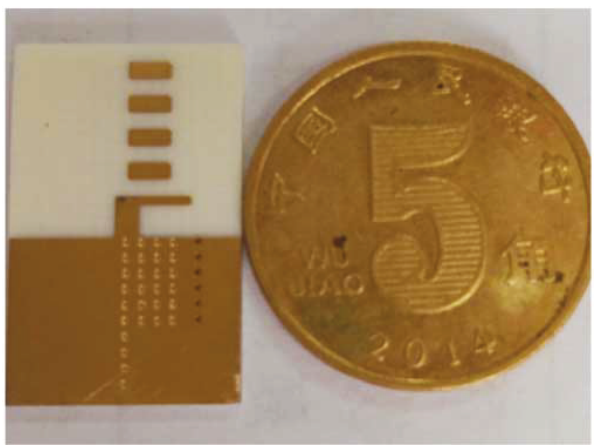

(b)

Figure 6: Photos of the fabricated slow-wave SIW Yagi antenna fabricated by Rogers 4350 substrate. (a) Top view; (b) bottom view.

a truncated ground plane as the other layer of the SWHMSIW, the second arm of the dipole antenna, and four director elements printed in the opposite direction. In order to reduce the design parameters, all of the width, length, and space between the director elements are set up equal to each other. There are four rows of metallized vias designed in the sublayer dielectric substrate with top suspended. The occupying area of the proposed antenna is only $21.1 \times 13.4 \mathrm{~mm}^{2}$. To feed the antenna with a subminiature version A (SMA) connector, a microstrip line with width $\left(W_{5}\right)$ of 1.4 millimeters is designed. All values of the geometry parameters for the proposed Yagi antenna have been illustrated in Table 2 .

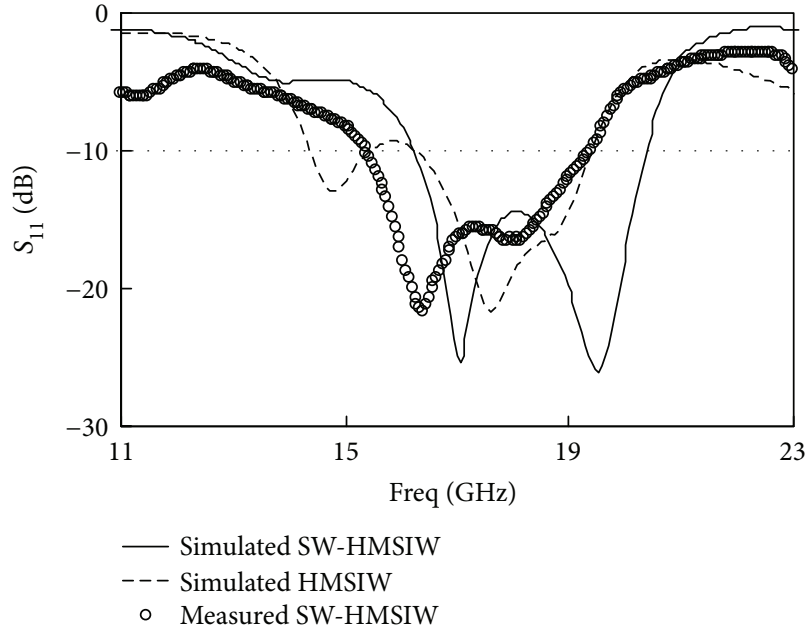

FIgURE 7: The comparison of $\left|S_{11}\right|$ for the computed HMSIW, computed SW-HMSIW, and measured SW-HMSIW-fed Yagi antennas.

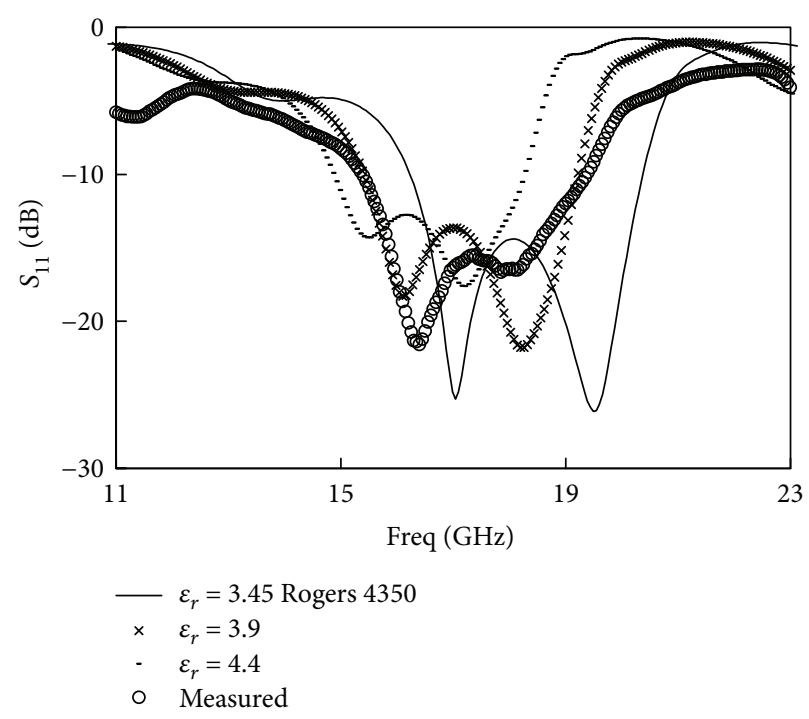

FIgURE 8: Computed $S_{11}$ values for the proposed metamaterial antennas with different relative permittivity.

\section{Electromagnetic Simulations and Experimental Results}

The proposed SW-HMSIW-fed Yagi antenna has been simulated, physically fabricated, and practically measured. The computation of the proposed antenna is done by Ansoft's HFSS software, and the measurement of $S$-parameters is made out by the Agilent vector network analyzer N5227A. The photos of the fabricated antenna with Rogers 4350 substrate have been shown in Figure 6. Figure 7 shows the comparison of $\left|S_{11}\right|$ for the computed HMSIW, computed SW-HMSIW, and measured SW-HMSIW-fed Yagi antennas. According to the $-10 \mathrm{~dB}$ level line, the simulated bandwidth of the HMSIW-fed antennas is from 


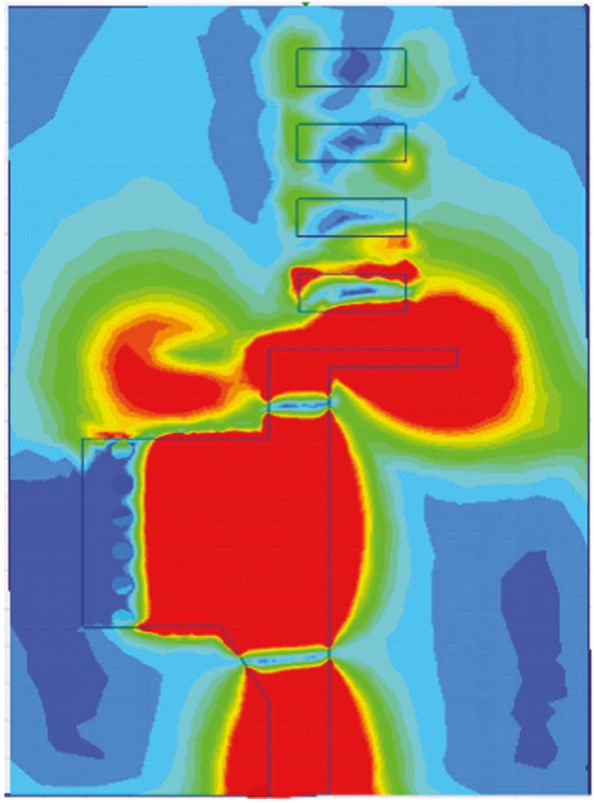

(a)

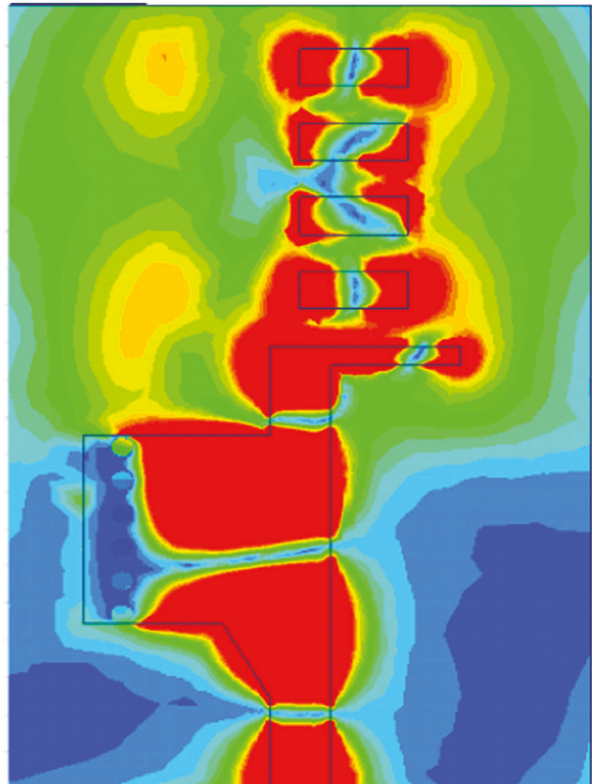

(b)

Figure 9: Simulated surface current distribution of proposed Yagi antenna at different frequencies: (a) $12 \mathrm{GHz}$; (b) $18 \mathrm{GHz}$.

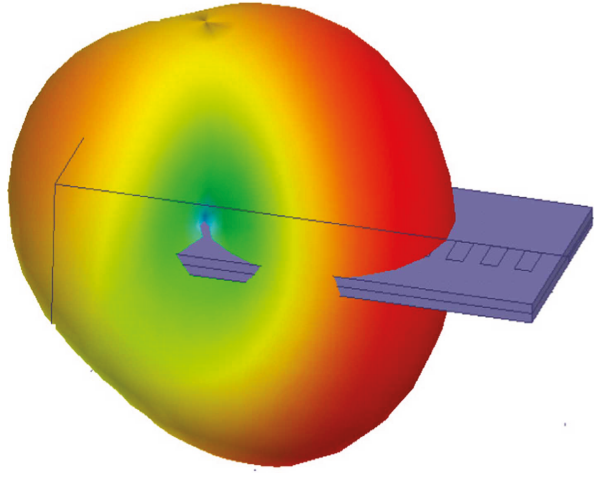

(a)

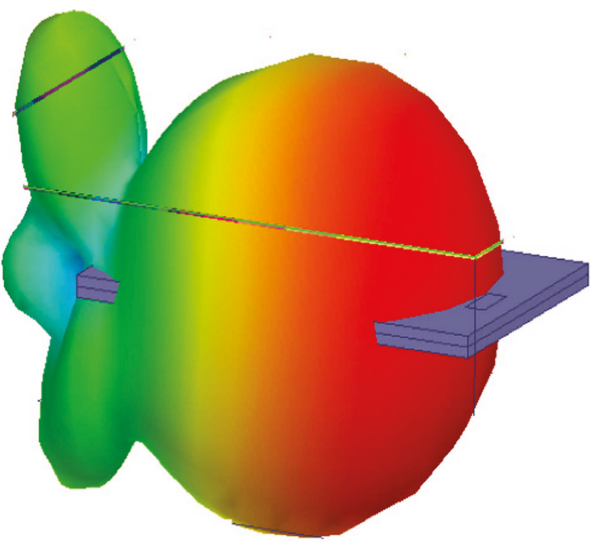

(b)

FiguRE 10: 3-D radiation patterns of the proposed Yagi antenna designed on Rogers 4350 substrate at different frequencies: (a) 12 GHz; (b) $18 \mathrm{GHz}$.

16.24 GHz to $19.3 \mathrm{GHz}$ with absolute bandwidth $3.06 \mathrm{GHz}$, the simulated SW-HMSIW antennas is from $16.2 \mathrm{GHz}$ to $20.3 \mathrm{GHz}$ with absolute bandwidth $4.1 \mathrm{GHz}$, and the measured bandwidth is from $15.4 \mathrm{GHz}$ to $19.4 \mathrm{GHz}$ with absolute bandwidth $4.0 \mathrm{GHz}$. As can be seen, compared to the conventional HMSIW-fed Yagi antenna, the proposed antenna has better impedance matching performance. A reasonable agreement between the simulations and measurements has been found convincing that the proposed antennas have reliable applications for $\mathrm{Ku}$ band wireless communications. To further analyze the deviation of $S_{11}$ between the experiment and simulation, the computed $S_{11}$ values of the antennas with different relative permittivity have been shown in Figure 8. It is obvious that the deviation of $S_{11}$ is mainly caused by the deviation dielectric constant.
The simulated surface current distribution of proposed SW-HMSIW-fed antenna at different frequencies $(12 \mathrm{GHz}$ and $18 \mathrm{GHz}$ ) has been shown in Figure 9. As can be illustrated, the maximum surface current is concentrated in the bottom side of the Yagi antenna at nonworking frequency band $(12 \mathrm{GHz})$. While at the operating frequency $(18 \mathrm{GHz})$, the distribution of surface current is more uniform. The $3-\mathrm{D}$ radiation patterns of the proposed antennas at $12 \mathrm{GHz}$ and $18 \mathrm{GHz}$ have been shown in Figure 10. It can be observed that the main beam of the proposed antennas becomes in the end-fire direction in the operating bandwidth.

The measured and simulated 2-D radiation patterns of the proposed Yagi antenna at different frequencies have been shown in Figure 11. The variations of simulated and measured peak-realized gain $(\mathrm{dBi})$ and radiation efficiency with frequency for the proposed antenna have been shown in 


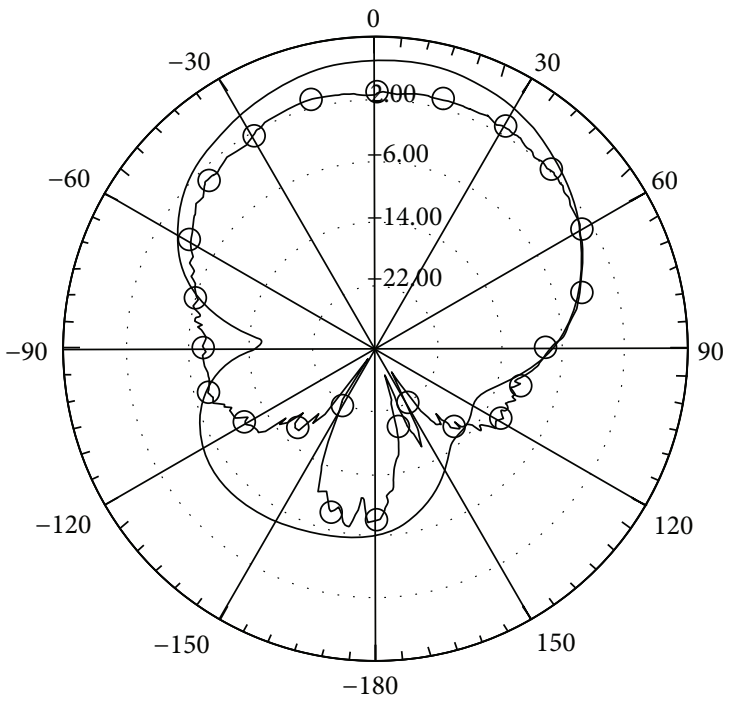

(a)

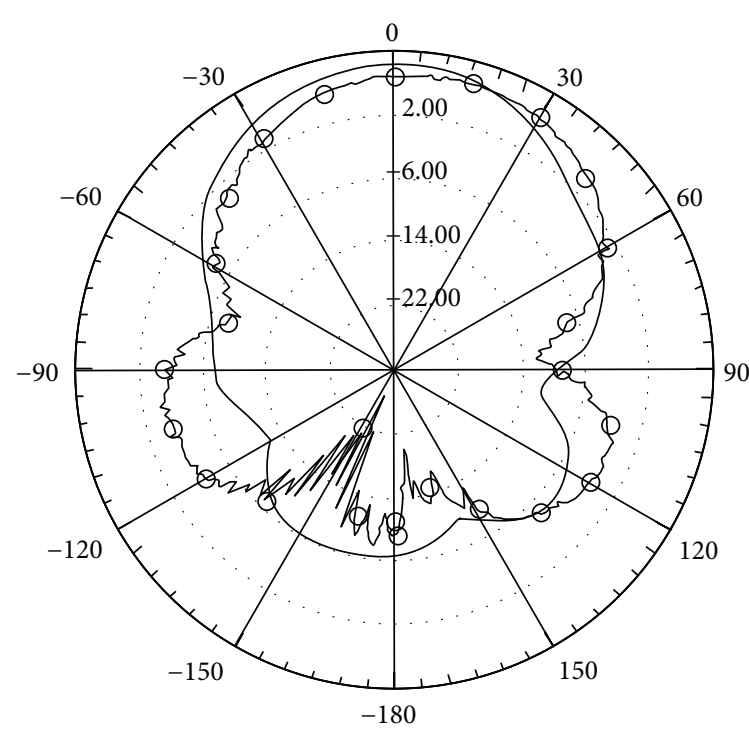

(b)

FIGURE 11: Measured and simulated 2-D radiation patterns of the proposed Yagi antenna at different frequencies (solid line: simulated data; line with circle symbol: measured data): (a) $15 \mathrm{GHz}$; (b) $18 \mathrm{GHz}$.

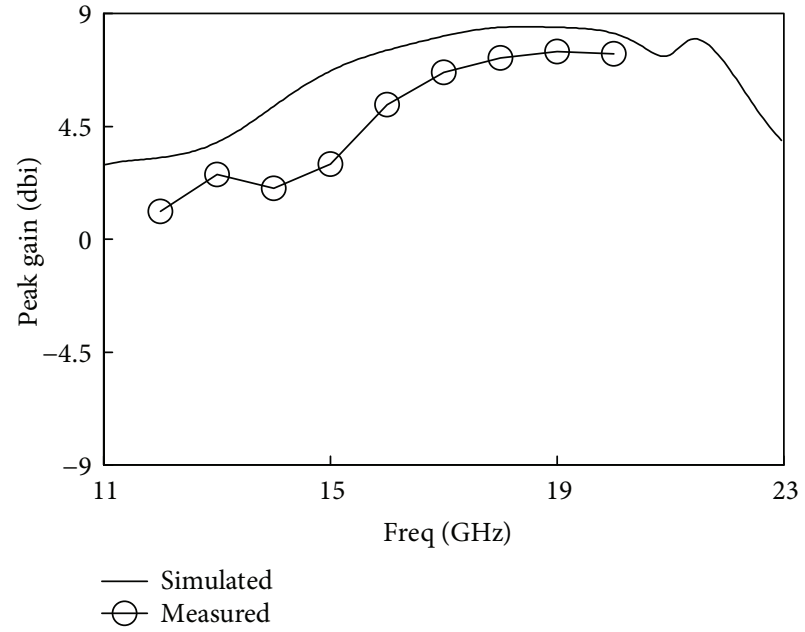

(a)

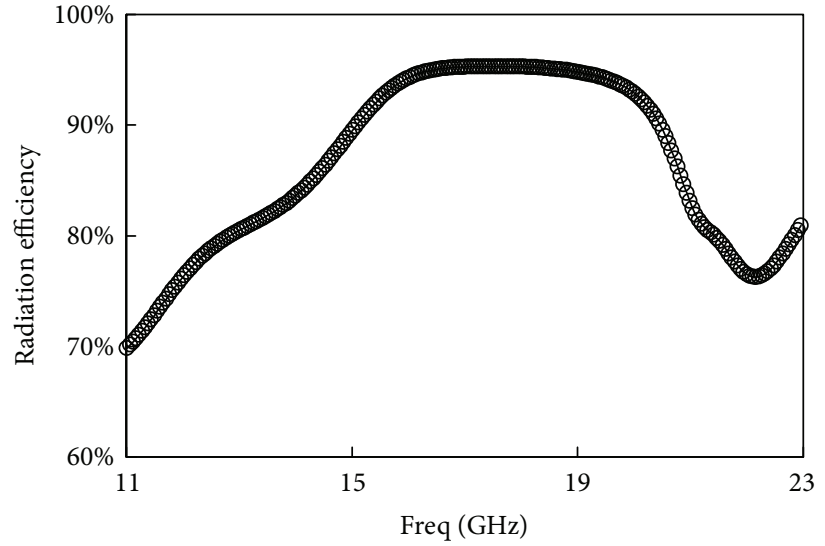

(b)

FIGURE 12: Variation of peak-realized gain $(\mathrm{dBi})$ and radiation efficiency with frequency: (a) peak-realized gain; (b) radiation efficiency.

Figure 12. The average simulated and measured gains of the proposed antennas are $8.1 \mathrm{dBi}$ (with the peak of $8.5 \mathrm{dBi}$ at $19.2 \mathrm{GHz}$ ) and 5.25 (with the peak of $7.49 \mathrm{dBi}$ at $19 \mathrm{GHz}$ ), respectively. The comparison of antenna performance for different feeding techniques has been shown in Table 3. It is obvious that the characteristics of the high gain, broadband, low insertion loss, and simple design for the proposed Yagi antenna have been verified by the simulated and measured results.

\section{Summary and Conclusions}

In this paper, the slow-wave HMSIW with Rogers 4350 substrate has been computed and studied. Compared with
TABle 3: The comparison of antenna performance for different feeding techniques.

\begin{tabular}{lccc}
\hline Feeding technique & Size & Bandwidth & Gain \\
\hline Microstrip-to-CPS balun & Poor & Medium & Medium \\
HMSIW & Medium & Medium & Medium \\
SW-HMSIW & Good & Good & Medium \\
\hline
\end{tabular}

the classical SIW, the SW-HMSIW can reduce the longitudinal dimension as the phase velocity is significantly smaller than the SIW structure. A novel SW-HMSIW-fed Yagi antenna has been simulated, physically fabricated, and practically measured. Good characteristics of the Yagi antenna, such as reduced size, high gain, broadband, and 
low insertion loss, have been verified by the computation and experiment. Measured results indicate that the impedance bandwidth of the proposed antenna is from $15.4 \mathrm{GHz}$ to $19.4 \mathrm{GHz}$ with the peak gain $7.49 \mathrm{dBi}$. The 3-D radiation patterns of the proposed antenna show that the main beam of the proposed antennas is in the end-fire direction in the operating bandwidth. All of these convince that the proposed antennas have reliable applications for $\mathrm{Ku}$ band wireless communications.

\section{Conflicts of Interest}

The authors declare that they have no conflicts of interest.

\section{Acknowledgments}

This study is supported in part by the National Natural Science Foundation of China (nos. 61401101 and 61672006), the Anhui Provincial Natural Science Foundation (nos. 1708085MF155 and 1608085QF159), the Natural Science Key Foundation of Anhui Provincial Universities (no. KJ2017A333), and the Anhui Provincial Outstanding Top-notch Talent Cultivation Project (gxfxZD2016164).

\section{References}

[1] N. Kaneda, Y. Qian, and T. Itoh, "A broad-band microstrip-towaveguide transition using quasi-Yagi antenna," IEEE Transactions on Microwave Theory and Techniques, vol. 47, no. 12, pp. 2562-2567, 1999.

[2] N. Kaneda, W. R. Deal, Y. Qian, R. Waterhouse, and T. Itoh, "A broadband planar quasi-Yagi antenna," IEEE Transactions on Antennas and Propagation, vol. 50, no. 8, pp. 1158-1160, 2002.

[3] D. Deslandes and K. Wu, "Integrated microstrip and rectangular waveguide in planar form," IEEE Microwave and Wireless Components Letters, vol. 11, no. 2, pp. 68-70, 200.

[4] M. Bozzi, "Substrate integrated waveguide (SIW): an emerging technology for wireless systems," in 2012 Asia Pacific Microwave Conference Proceedings, Kaohsiung, Taiwan, 2012.

[5] N. Grigoropoulos, B. S. Izquierdo, and P. R. Young, "Substrate integrated folded waveguides (SIFW) and filters," IEEE Microwave and Wireless Components Letters, vol. 15, no. 12, pp. 829-831, 2005

[6] B. Liu, W. Hong, Y. Q. Wang, Q. H. Lai, and K. Wu, "Half mode substrate integrated waveguide (HMSIW) 3-dB coupler," IEEE Microwave and Wireless Components Letters, vol. 17, no. 1, pp. 22-24, 2007.

[7] F. Farzami and M. Norooziarab, "Experimental realization of tunable transmission lines based on single-layer SIWs loaded by embedded SRRs," IEEE Transactions on Microwave Theory and Techniques, vol. 61, no. 8, pp. 2848-2857, 2013.

[8] F. Farzami, K. Forooraghi, and M. Norooziarab, "Design and modeling of a miniaturized substrate integrated waveguide using embedded SRRs," IEEE Antennas and Wireless Propagation Letters, vol. 10, pp. 713-716, 2011.

[9] X. Zou, C.-M. Tong, J.-S. Bao, and W.-J. Pang, "SIW-fed Yagi antenna and its application on monopulse antenna," IEEE Antennas and Wireless Propagation Letters, vol. 13, pp. 1035-1038, 2014.
[10] G. Zhai, W. Hong, K. Wu, and Z. Kuai, "Printed quasi-Yagi antenna fed by half mode substrate integrated waveguide," 2008 Asia-Pacific Microwave Conference, 2008, pp. 1-4, Hong Kong, China, 2008.

[11] A. Niembro-Martin, V. Nasserddine, E. Pistono et al., "Slowwave substrate integrated waveguide," IEEE Transactions on Microwave Theory and Techniques, vol. 62, no. 8, pp. 16251633, 2014.

[12] HFSS, “Ansys, Canonsburg, PA, USA," 2014, http://www. ansys.com/products/electronics/ansys-hfss. 


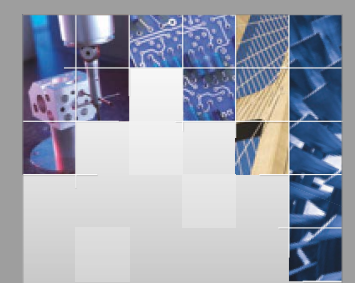

\section{Enfincering}
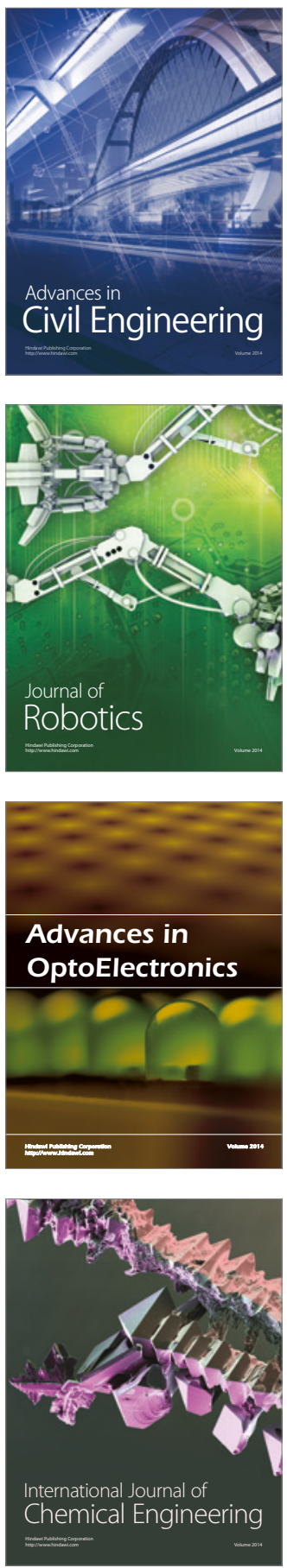

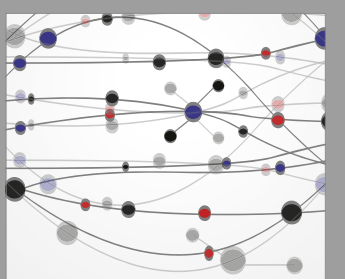

The Scientific World Journal

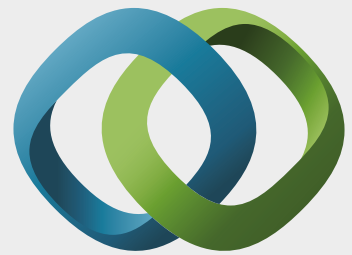

\section{Hindawi}

Submit your manuscripts at

https://www.hindawi.com
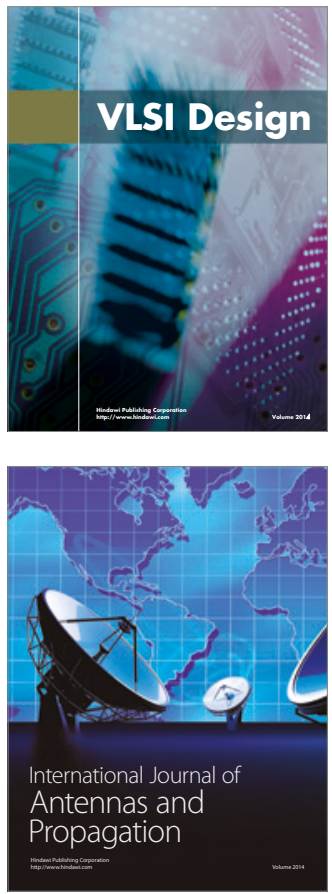

\section{Rotating}

Machinery
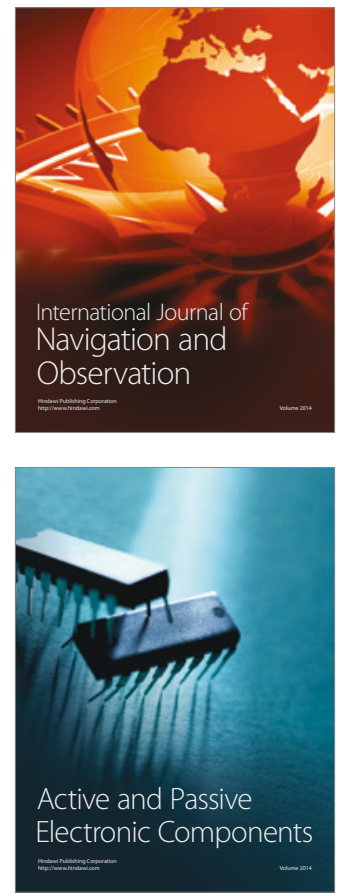
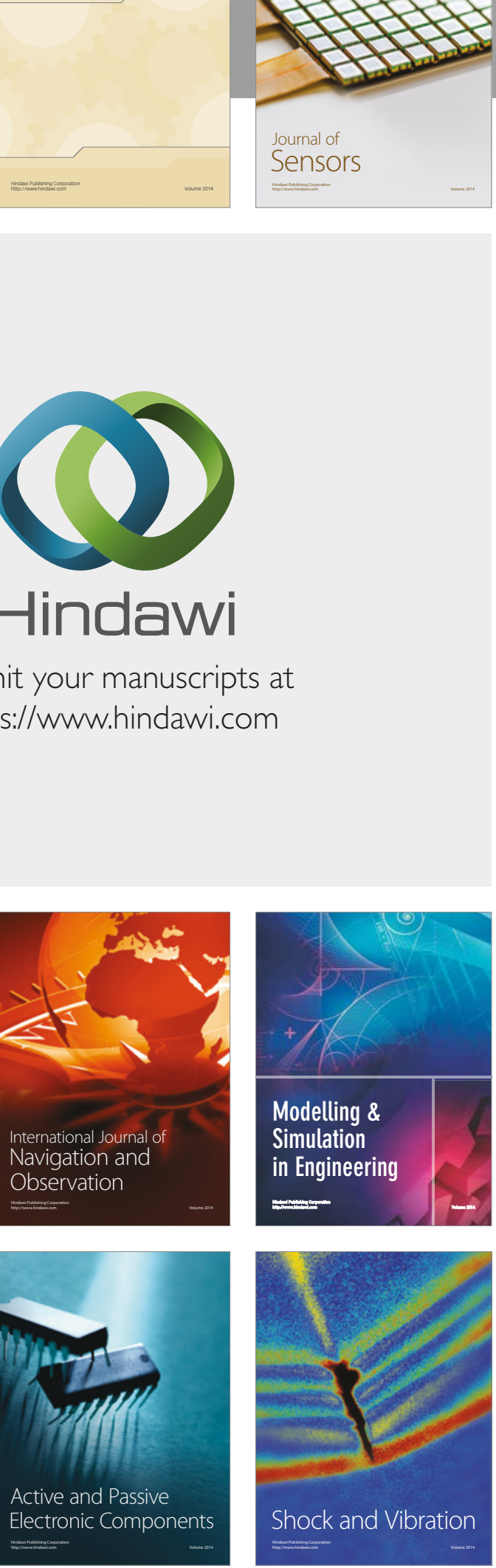
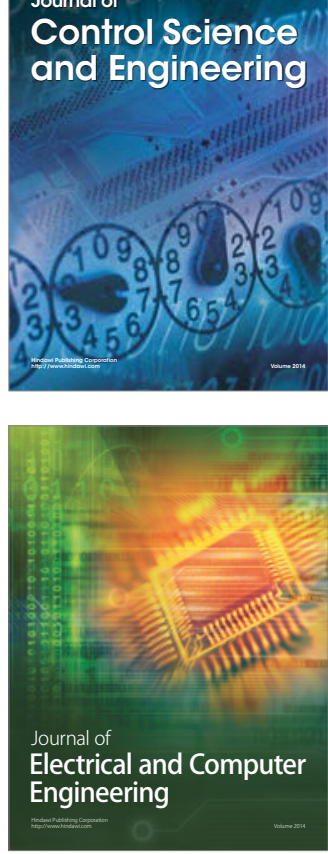

Distributed

Journal of

Control Science

and Engineering
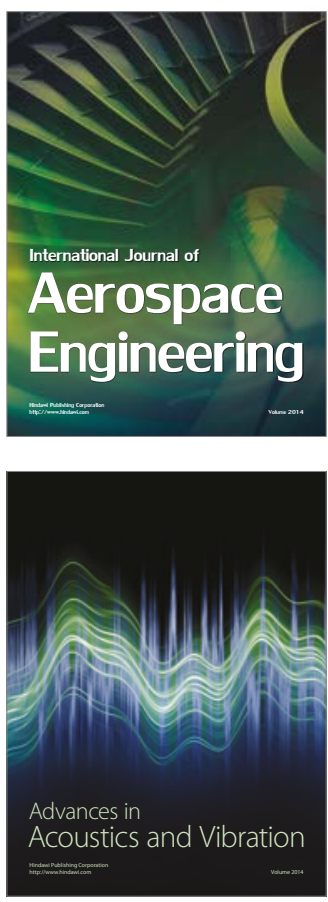

Sensor Networks 